\title{
Constrainedly Fair Job Assignments under Minimum Wages
}

\author{
T. Andersson ${ }^{\mathrm{a}, *}$, L-G. Svensson ${ }^{\mathrm{a}}$, Z. Yang ${ }^{\mathrm{b}}$ \\ ${ }^{a}$ Department of Economics, Lund University, P.O. Box 7082, SE-222 07 Lund, Sweden \\ ${ }^{b}$ Faculty of Business Administration, Yokohama National University, Yokohama 240-8501, Japan
}

\begin{abstract}
A number of jobs are to be assigned to a greater number of workers. While having to abide by the law of minimum wages, firms must determine who should be assigned which job and at what salary. In such situations fair (envy-free) allocations usually fail to exist. To cope with this situation, this paper proposes a new concept of fairness, called constrained fairness. Among the set of constrainedly fair allocations, the so-called constrainedly fair and minimal allocations are of particular interest. The reason for this is that the salaries are not only compatible with the minimum wages; they are also optimal from the standpoint of cost-minimizing firms and the assignment of jobs is efficient from the viewpoint of workers. A convergent dynamic procedure that identifies constrainedly fair and minimal allocations is proposed. Furthermore, strategic properties of the mechanism are derived, and two notions of efficiency are evaluated.
\end{abstract}

Key words: Minimum wages, job assignment, constrained fairness, dynamic mechanism design, incentives, non-manipulability.

JEL Classification: C78, D02, D63, J33

\section{Introduction}

A minimum wage refers to a minimum (hourly, monthly etc.) amount which employers must pay their employees for their work and service. One of the main objectives with a minimum wage law is to protect employees from being exploited, i.e., to ensure that employees do not receive too low salaries. However, it is well-known that disequilibrium situations may arise when minimum wage laws are enacted, i.e., for a given set of wages it may not be possible to assign each worker his/her most preferred job. Consequently, analyzing job-assignment problems with minimum wage restrictions requires a new approach beyond the existing ones for the traditional fair (or envy-free) job-assignment problems investigated by e.g., Alkan et al. (1991), Crawford and Knoer (1981), Maskin (1987), Svensson (1983) and Tadenuma and Thomson (1991), where each worker is assigned his most desired job at the given wages, simply because such an allocation may not be attainable under the minimum wage requirement. This paper investigates a job-assignment

\footnotetext{
The authors would like to thank the editor and anonymous referees for their detailed and helpful comments and criticism. We have also received helpful comments from seminar participants at Uppsala University and the Society for Economic Design meeting (Maastricht, 2009). Andersson and Svensson acknowledge financial support from the Tom Hedelius and Jan Wallander foundation, and Yang is supported by the Ministry of Education, Science, and Technology of Japan.

${ }^{*}$ Corresponding author

Email addresses: tommy. andersson@nek.lu.se (T. Andersson), lars-gunnar.svensson@nek.lu.se (L-G. Svensson), yang@ynu.ac.jp (Z. Yang)
} 
problem and proposes a weakening of the fairness concept 1 which is tailored to capture the specific features of labor markets with exogenously given minimum wages and unemployment benefit.

The job assignment model consists of a finite number of jobs and a greater number of workers. Each worker is permitted to have at most one job, but some workers will be unemployed (since there are more workers than jobs). The employer specifies a wage for each job under a minimum wage constraint, meaning that any worker who is assigned a job must be paid no less than its corresponding minimum wage. A worker that is not assigned a job receives an unemployment benefit. The level of the unemployment benefit and the minimum wages are set by the government and are regarded as fixed in the model. For this type of job assignment model there are (at least) two compelling reasons for abandoning the standard fair job assignment approach. First, there may not exist a fair allocation that satisfies the wage requirements (see, e.g., Example (1). Second, in the real world, many unemployed workers would like to have a job and are therefore willing to accept a job at a lower wage rather than being unemployed. Thus by adopting the notion of fairness some wages may fall short of the minimum wages and this cannot be the case. For these reasons the concept of fairness must be weakened. This paper proposes a weakening called constrained fairness where the unemployed workers are allowed to envy employed workers but no employed worker is allowed to envy any other worker (employed or unemployed). This weakening captures the facts that (i) unemployed workers may like to be employed, (ii) that accepting a job offer is a voluntary decision (because each employed worker is assigned his most preferred job, at the given wages, and the worker finds this to be a better alternative than being unemployed) and (iii) the economic reality that firms are only responsible for their employees and can therefore only guarantee an equilibrium outcome among their employees.

The first major result (Theorem 1) demonstrates under very mild conditions that the set of compensation vectors (or wage vectors) that respect the constrained fairness criterion is non-empty and, moreover, that there exists a unique compensation vector that is minimal in the sense that it requires the employer to pay the least. This vector is called the constrainedly fair and minimal compensation vector and it is of particular interest not only because it minimizes the wage costs for the employer but also because the (possible) envy is minimized for the specific job assignment. More precisely, because constrainedly fair allocations cannot exclude the possibility that an unemployed worker envies an employed worker, it is desirable to reduce such envy as much as possible 2 Another motivation for considering this particular wage vector is that there is no reason for a cost minimizing firm to require the wages to be higher than necessary. The existence of constrainedly fair and minimal job-allocations is established in Theorem 2

Given the existence of constrainedly fair and minimal allocations, it is of interest to investigate strategic properties of a general allocation mechanism that always selects a constrainedly fair and minimal allocation. Such a mechanism is called the constrained fair and minimal allocation (CFMA) mechanism and has several interesting strategic features. Unlike e.g., the Vickrey-Clarke-Groves mechanism, the CFMA mechanism does not have an equilibrium in dominant strategies for all workers in every situation. However, Theorems 3 and4/demonstrate (under very general circumstances) that the CFMA mechanism neither can be manipulated by any group of unemployed workers when the unemployment benefit is high relative to the minimum wages, nor can it be manipulated by any group of employed workers when the minimum wages are high relative to the unemployment benefit. These results are quite original and surprisingly consistent with what is often observed in the real world. For instance, when the unemployment benefit is reasonably high, unemployed workers are generally contented with the situation and in fact have no incentive to apply for jobs at all (thus no manipulation).

\footnotetext{
${ }^{1}$ Andersson and Svensson (2008) and Diamantaras and Thomson (1990) suggest alternative weakenings.

${ }^{2}$ Chaudhuri (1986) presents an intensity measure of envy from a different perspective.
} 
Another important issue is to design a simple and practical procedure for identifying constrainedly fair and minimal allocations. This paper develops a dynamic procedure (Algorithm 1) and demonstrates that it indeed converges in a finite number of steps and in addition identifies the constrainedly fair and minimal compensation vector (Theorem 5) for the quasi-linear utilities. The proposed algorithm is a dynamic salary adjustment procedure that starts with the exogenously given minimum wages. The algorithm then increases the wages for the well-defined so-called over-supplied jobs in each iteration until it converges (formally a set of jobs is over-supplied if the number of workers who demand a job in the set is less than the number of jobs in the set). This dynamic procedure is a variant of the Hungarian method of Kuhn (1955) in the sense that it starts with a well-defined matrix (in this case each element in the matrix represents the valuation of a specific worker of being assigned a certain job and its corresponding wage) and then adjusts this matrix by systematically changing the wages until a constrainedly fair compensation vector can be identified. While several related algorithms in the literature adjust the wages for over-demanded jobs (i.e., a set of jobs that are demanded by a larger number of workers than there are jobs in the set), see, e.g., Crawford and Knoer (1981) or Demange et al. (1986), our algorithm adjusts the wages for "over-supplied" jobs. In fact, we cannot adjust wages for over-demanded jobs, because jobs may always be over-demanded due to the lower compensation limits. It should also be noted that the current algorithm is different from those proposed by Su (1999), Klijn (2000), Brams and Kilgour (2001), and Haake et al. (2002), because they all require that a fixed (possibly non-negative) amount of money should be completely allocated among the agents. Hence, no such minimal compensation scheme exists in their models.

This paper also examines the problem of efficiently assigning the jobs to the workers given the unique constrainedly fair and minimal compensation vector. At a first sight this is an easy problem given the information generated in the final step of our algorithm because this step essentially reveals which objects are preferred by each worker at the constrainedly fair and minimal compensations. However, by only using this information there is a risk that a Pareto dominated job-assignment is selected 3 We solve this problem by providing a concrete procedure for identifying an efficient job assignment that is compatible with the (unique) constrainedly fair and minimal compensation vector (Algorithm 2).

The paper ends with a discussion on the social desirability of constrainedly fair and minimal allocations, and reveals that such allocations are optimal for the firms but may not always coincide with the goal of a social planner. In particular, given quasi-linear utilities, it is demonstrated that a constrainedly fair and minimal allocation need not be welfare efficient in the sense that it need not maximize the sum of the values of the jobs to the workers. In this sense there is a potential trade-off between constrainedly fair and minimal compensations and welfare efficiency. However, if the employer (for some reason) attaches more importance to welfare efficiency, we show that the dynamic procedures can be modified to reach a socially desirable allocation which is both welfare efficient and constrained fair (Algorithm 3).

The paper is outlined as follows. In Section 2 the formal job-assignment model is presented. Section 3 introduces and defines the concept of constrained fairness. Section 4 investigates a number of properties of constrainedly fair and minimal allocations. Section 5 derives several strategic properties of the CFMA mechanism. Section 6 specifies two algorithms that together identify constrainedly fair and minimal allocations. Finally, Section 7 discusses the social desirability of constrainedly fair and minimal allocations.

\footnotetext{
${ }^{3}$ This is not a problem in traditional fair job-assignment models because the set of fair allocations is a subset of the set of the Pareto-efficient allocations see, e.g., Svensson (1983).
} 


\section{The Job Assignment Model}

The job assignment model consists of $m$ workers and $n$ jobs. The finite sets of workers and jobs are denoted by $M=\{1, \ldots, m\}$ and $N=\{1, \ldots, n\}$, respectively. It is supposed that $m>n$, i.e., at least one worker will be unemployed. For convenience, each unemployed worker is assigned a dummy-job denoted by $0 \notin N$. The wage for job $j \in N$ is given by $s_{j}$, and all wages are collected in a compensation vector $s=\left(s_{1}, \ldots, s_{n}\right)$. An unemployed worker receives a fixed unemployment benefit $s_{0}$ from the government. The government also determines a minimum wage $w_{j}$ for each job $j \in N$. The minimum wages are gathered in the vector $w=\left(w_{1}, \ldots, w_{n}\right)$. This means that $s_{j} \geq w_{j}$ for all $j \in N$. Note also that because the unemployment benefit is fixed it is always equal to $s_{0}$ so there is no reason to introduce a minimum wage for the dummy-job.

Each worker demands at most one job and the utility for worker $i \in M$ of being assigned job $j \in N \cup\{0\}$ with compensation $s_{j}$ is given by $u_{i j}\left(s_{j}\right)$ where the utility function is continuous and strictly increasing in $s_{j}$. It is also assumed that no job is infinitely good or bad for any worker. This assumption guarantees that for any worker and any two jobs there are wages that make the worker indifferent between the two jobs. A list $u=\left(u_{1}, \ldots, u_{m}\right)$ of (individual) utility functions is a (preference) profile. We also adopt the notational convention of writing $u=\left(u_{C}, u_{-C}\right)$ for $C \subset N$. The set of profiles with utility functions having the above properties is denoted by $\mathcal{U}$.

An assignment is a mapping $\pi: M \mapsto N \cup\{0\}$ assigning job $\pi_{i}$ to worker $i \in M$. An assignment $\pi$ is feasible if $\pi$ is onto and $\pi_{i}=\pi_{k}$ and $i \neq k$ implies that $\pi_{i}=0$. Hence, any real job is assigned to precisely one worker. An allocation $(\pi, s)$ consists of a compensation vector $s$ and a feasible assignment $\pi$. The set of allocations is denoted by $\mathcal{A}$. An allocation mechanism is a non-empty correspondence $\varphi$ that for each profile $u \in \mathcal{U}$ selects a set of allocations $\varphi(u) \subset \mathcal{A}$ such that $u_{i \pi_{i}}\left(s_{\pi_{i}}\right)=u_{i \hat{\pi}_{i}}\left(\hat{s}_{\hat{\pi}_{i}}\right)$ for all $i \in M$ if $\left(\pi_{i}, s\right) \in \varphi(u)$ and $(\hat{\pi}, \hat{s}) \in \varphi(u)$. Hence, the various outcomes in the set $\varphi(u)$ are utility equivalent, and such a correspondence is called essentially single-valued (ESV).

\section{Constrained fairness}

In the classical definition of fairness (due to Foley, 1967) an allocation is fair if it is envy-free, i.e., allocation $(\pi, s)$ is fair if $\pi$ is feasible and $u_{i \pi_{i}}\left(s_{\pi_{i}}\right) \geq u_{i j}\left(s_{j}\right)$ for all $i \in M$ and all $j \in N \cup\{0\}$. However, the set of allowable fair allocations may be empty in our job assignment model because monetary restrictions are imposed on the unemployment benefit and the wages. This is illustrated in the following example.

Example 1. Suppose that $M=\{1,2\}$ and $N=\{1\}$ so one of the workers will be unemployed. Assume also that the workers are endowed with quasi-linear utility functions of the type $u_{i j}\left(s_{j}\right)=v_{i j}+s_{j}$ for $i \in M$ and $j \in N$, and let $v_{10}=v_{20}=3$ and $v_{11}=v_{21}=2$. Clearly both workers strictly prefer to be employed when $s_{1}-s_{0}>1$. Say now that the government announces that the unemployment benefit $s_{0}$ equals 1 and that the minimum wage for job 1 is set to $w_{1}$. Then, because $s_{0}=1$ is fixed and $s_{1} \geq w_{1}$ by law, for any $w_{1}>2$ the unemployed worker will always envy the employed worker and thus fair allocations cannot be achieved.

Due to the problems illustrated in Example 1, the concept of fairness must be modified. The following notion is adopted in this paper.

Definition 1. For a given profile $u \in \mathcal{U}$, allocation $(\pi, s)$ is said to be constrainedly fair (CF, henceforth) if

(i) $s \geq w$,

(ii) $\pi$ is feasible, 
(iii) $u_{i \pi_{i}}\left(s_{\pi_{i}}\right) \geq u_{i j}\left(s_{j}\right)$ for all $i \in M$ with $\pi_{i} \neq 0$ and all $j \in N \cup\{0\}$.

If $(\pi, s)$ is a CF allocation, then $s$ is called a CF compensation vector and $\pi$ is said to be compatible with $s . A$ compensation vector $s$ is $C F$ if there is an assignment $\pi$ such that $(\pi, s)$ is a CF allocation. All compensation vectors that are $C F$ for a given profile $u \in \mathcal{U}$ are collected in the set $F(u)$.

The meaning of the above definition is that an allocation is CF if the wages are weakly higher than the minimum wages and if each employed worker is allocated his most preferred job at the given wages. Thus, no employed worker envies any other worker (employed or unemployed), but an unemployed worker may envy an employed worker 4 An unemployed worker will, however, never envy any other unemployed worker because the unemployment benefit (i.e., the salary for the dummy-job $s_{0}$ ) is fixed and equal for all unemployed workers. Note also that a fair allocation is always CF. In this sense, the notion of CF is a weakening of the fairness concept. Moreover, in the bounding case (not considered in this paper) when $|N|=|M|$ the two notions coincide 5

We end this section with an important result from the fairness literature that will be adopted in several of the coming proofs. The result is a direct consequence of the Perturbation Lemma in Alkan et al. (1991) and is stated here without a proof.

Lemma 1. If no worker in $M^{\prime} \subseteq M$ envies any other worker in $M^{\prime}$ at allocation $(\pi, s)$, then, for each $\varepsilon>0$, there exists some other allocation $(\hat{\pi}, \hat{s})$ where no worker in $M^{\prime}$ envies any other worker in $M^{\prime}, \hat{\pi}_{i}=\pi_{i}$ for all $i \in M-M^{\prime}$, and $\hat{s}_{\pi_{j}}<s_{\pi_{j}}<\hat{s}_{\pi_{j}}+\varepsilon$ for all $j \in M^{\prime}$.

\section{Constrainedly Fair and Minimal Allocations}

Constrained fairness concerns primarily employed workers. The compensation vector is required to define an equilibrium among employed workers but not necessarily among unemployed workers. As observed in the previous section, it may happen that an unemployed worker envies an employed worker. This potential disequilibrium situation is a necessary consequence of the restrictions on the unemployment benefit and the wages (imposed by the government). One reason to deviate from equilibrium, by putting lower limits on the wages, is that the market solution (i.e., the fair solution) from a social point of view may yield too low compensations. For example, one of the main arguments for introducing minimum wage laws is to protect the employees from receiving too low salaries. However, if a lower limit is imposed, there is no reason for a cost-minimizing employer to pay higher compensations than the market demands. Hence, of primary interest is to identify a CF compensation vector $s^{*}$ with the property that wage for each job in $s^{*}$ is smaller than or equal to the wage for the same job at any CF compensation vector. Such a compensation vector is called $\mathrm{CF}$ and minimal.

Definition 2. For a given profile $u \in \mathcal{U}$, a compensation vector $s^{*} \in F(u)$ is said to be $C F$ and minimal if $s_{j}^{*} \leq s_{j}$ for all $j \in N$ and all $s \in F(u)$.

\footnotetext{
${ }^{4}$ Given the setup in Example 1, it is clear that any allocation $(s, \pi)$ where $s_{0}=1$ and $s_{1} \geq 2$ is CF as long as $\pi$ is feasible. The unemployed worker will, however, envy the employed worker when $s_{1}$ exceeds 2 .

${ }^{5}$ Clearly, fairness implies CF. Suppose now that allocation $(\pi, s)$ is CF and that $|N|=|M|$. From the feasibility requirement it then follows that $j=\pi_{i}$ for some $i \in M$ for each $j \in N$. Hence, $\pi_{i} \neq 0$ for all $i \in M$ since $|N|=|M|$. But then $u_{i \pi_{i}}\left(s_{\pi_{i}}\right) \geq u_{i j}\left(s_{j}\right)$ for all $i \in M$ and all $j \in N \cup\{0\}$ by definition of CF. Thus allocation $(\pi, s)$ is also fair.
} 
Before establishing the existence of a $\mathrm{CF}$ and minimal compensation vector some notation must be introduced. Let $T$ be a subset of jobs and denote by $M(s, T)$ the set of workers who demand a job in $T$ at the compensation vector $s$, i.e.,

$$
M(s, T)=\left\{i \in M \mid u_{i j}\left(s_{j}\right)=\max _{k \in N \cup\{0\}} u_{i k}\left(s_{k}\right) \text { for some } j \in T\right\} .
$$

A well-known result by Hall (1935) states that if there are exactly as many workers as there are jobs, then it is possible to fairly assign a job to each worker if and only if for every set $T$ of jobs, the number of workers demanding jobs from $T$ is at least as large as the number of elements in $T$, i.e., if and only if $|T| \leq|M(s, T)|$ for all $T \subseteq N$. Lemma 2 (below) applies Hall's Theorem to prove a corresponding property of CF compensation vectors. This lemma is then useful in establishing the existence and the uniqueness of a $\mathrm{CF}$ and minimal compensation vector.

Lemma 2. For each profile $u \in \mathcal{U}$, a compensation vector $s$ belongs to $F(u)$ if and only if $|T| \leq|M(s, T)|$ for all $T \subseteq N$.

Proof. Let $u \in \mathcal{U}$. Clearly $|T| \leq|M(s, T)|$ for all $T \subseteq N$ if $s \in F(u)$. Suppose now that $|T| \leq|M(s, T)|$ for all $T \subseteq N$. To prove that $s \in F(u)$, consider $N^{\prime}=N \cup\{m+1, \ldots, n\}$, where each $j$ in $N^{\prime}-N$ is one of $n-m$ dummy-jobs. Let also $v_{i}$ for $i \in M$ be utility functions defined as: $v_{i j}\left(s_{j}\right)=u_{i j}\left(s_{j}\right)$ if $j \in N$, and $v_{i j}\left(s_{j}\right)=\max _{k} u_{i k}\left(s_{k}\right)$ for $k \in N \cup\{0\}$ if $j \in N^{\prime}-N$. From the assumption $|T| \leq|M(s, T)|$ for all $T \subseteq N$ it then follows that:

$$
|T| \leq\left|M^{\prime}(s, T)\right| \text { for all } T \subseteq N^{\prime},
$$

where:

$$
M^{\prime}(s, T)=\left\{i \in M \mid v_{i j}\left(s_{j}\right)=\max _{k \in N^{\prime}} v_{i k}\left(s_{k}\right) \text { for some } j \in T\right\} .
$$

Note that $\left|M^{\prime}(s, T)\right|=|M(s, T)|$ if $T \subseteq N$ while $\left|M^{\prime}(s, T)\right|=m$ if $T \cap N^{\prime}-N \neq \varnothing$. But then according to Hall's Theorem, there is an assignment $\pi$ such that $(\pi, s)$ is a fair allocation at $v$ since $\left|N^{\prime}\right|=|M|$. Consequently, $(\tau, s)$ is CF at $u$ if $\tau_{i}=\pi_{i}$ when $\pi_{i} \leq m$ and $\tau_{i}=0$ when $\pi_{i}>m$. Thus, $s \in F(u)$.

Theorem 1. For each profile $u \in \mathcal{U}$, there exists a unique $C F$ and minimal compensation vector $s^{*}$.

Proof. We first prove that $F(u) \neq \varnothing$. Take an arbitrary subset $L$ of $n$ workers from $M$. For any real vector $y=\left(y_{1}, \cdots, y_{n}\right)$ there exist a real vector $x=\left(x_{1}, \cdots, x_{n}\right) \geq y$ and a bijective function $\beta: L \mapsto N-\{0\}$ such that $u_{i \beta_{i}}\left(x_{\beta_{i}}\right) \geq u_{i 0}(0)$ and $u_{i \beta_{i}}\left(x_{\beta_{i}}\right) \geq u_{i j}\left(x_{j}\right)$ for all $i \in L$ and all $j \in N-\{0\}$, by monotonicity, see, e.g., Maskin (1987) or Svensson (1983). Now it is readily seen that $F(u) \neq \varnothing$. For the same reason, $F(u)$ is not bounded above, but bounded below since $s \geq w$ for all $s \in F(u)$. Moreover, $F(u)$ is closed because preferences are continuous.

Now, it only remains to prove that $\underline{s} \in F(u)$ whenever $\underline{s}_{j}=\min \left\{s_{j}, s_{j}^{\prime}\right\}$ for all $j \in N$ and $s, s^{\prime} \in F(u)$. Then, because $F(u)$ is closed and bounded below, there is a unique minimal compensation vector. Consider two vectors $s, s^{\prime} \in F(u)$ and let $A=\left\{j \in N \mid s_{j}^{\prime}<s_{j}\right\}$. Without loss of generality, it is assumed that $A \neq N$ and $A \neq \varnothing$.

We first prove that there is a group $G \subseteq M$ of workers that can be fairly assigned to jobs in $A$. To obtain a contradiction, suppose that (a) there is a non-empty subset $T \subseteq A$ such that $|T|>|M(\underline{s}, T)|$. Consider next worker $i \in M\left(s^{\prime}, T\right)$ where $u_{i j}\left(s_{j}^{\prime}\right)=\max _{k \in N \cup\{0\}} u_{i k}\left(s_{k}^{\prime}\right)$ for some $j \in T \subseteq A$, by definition of $M\left(s^{\prime}, T\right)$. For this worker, it is clear that:

$$
u_{i j}\left(\underline{s}_{j}\right)=u_{i j}\left(s_{j}^{\prime}\right)=\max _{k \in N \cup\{0\}} u_{i k}\left(s_{k}^{\prime}\right) \geq \max _{k \in N \cup\{0\}} u_{i k}\left(\underline{s}_{k}\right) \text { for some } j \in T \subseteq A,
$$


by monotonicity. Hence, $M\left(s^{\prime}, T\right) \subseteq M(\underline{s}, T)$, so $|T|>|M(\underline{s}, T)| \geq\left|M\left(s^{\prime}, T\right)\right|$ by assumption (a). But since $s^{\prime} \in F(u)$, the latter condition contradicts Lemma 2. Hence, assumption (a) cannot be true. By applying Lemma2 2 once more, there is an allocation $(\sigma, \underline{s})$ such that $u_{i \sigma_{i}}\left(\underline{s}_{\sigma_{i}}\right) \geq u_{i j}\left(\underline{s}_{j}\right)$ for all $\sigma_{i} \in A$ and all $j \in N \cup\{0\}$. Let now $G=\left\{i \in M \mid \sigma_{i} \in A\right\}$.

We next prove that $\underline{s} \in F(u)$. Let allocation $(\pi, s)$ be CF, and consider a job $j \in N-A$ and the worker $i \in M$ with $\pi_{i}=j$. Now we have $u_{i \pi_{i}}\left(s_{\pi_{i}}\right) \geq u_{i k}\left(s_{k}\right)>u_{i k}\left(s_{k}^{\prime}\right)=u_{i k}\left(\underline{s}_{k}\right)$ for all $k \in A$, since $(\pi, s)$ is CF and preferences are monotonic. Moreover, $u_{i \pi_{i}}\left(\underline{s}_{\pi_{i}}\right)=u_{i \pi_{i}}\left(s_{\pi_{i}}\right)$ because $\pi_{i} \in N-A$ and, consequently, $u_{i \pi_{i}}(\underline{s})>u_{i k}(\underline{s})$. Hence, $i \neq G$. But then allocation $(\tau, \underline{s})$ is CF if $\tau_{i}=\sigma_{i}$ for $i \in G$ and $\tau_{i}=\pi_{i}$ for $i \in M-G$. Thus, $\underline{s} \in F(u)$.

Given the existence of a (unique) $\mathrm{CF}$ and minimal compensation vector $s^{*}$ it follows from the definition of CF that there exists an assignment $\pi$ that is compatible with $s^{*}$. More precisely, let $\hat{R}_{i}\left(s^{*}\right)$ be the set of jobs in $N \cup\{0\}$ that maximizes the utility of worker $i \in M$ at the CF and minimal compensation vector $s^{*}$, i.e.,

$$
\hat{R}_{i}\left(s^{*}\right)=\left\{j \in N \cup\{0\} \mid u_{i j}\left(s_{j}^{*}\right)=\max _{k \in N \cup\{0\}} u_{i k}\left(s_{k}^{*}\right)\right\} .
$$

By defining $R_{i}\left(s^{*}\right)=\hat{R}_{i}\left(s^{*}\right)-\{0\}$, the following result can be obtained.

Lemma 3. Any feasible assignment $\pi$ where $\pi_{i} \in R_{i}\left(s^{*}\right)$ for all $i \in M$ with $\pi_{i} \neq 0$ is compatible with $s^{*}$.

Proof. Note that if $\hat{R}_{i}\left(s^{*}\right)=\{0\}$ so $R_{i}\left(s^{*}\right)=\varnothing$ for some $i \in M$ then this worker can never be assigned a job from $N$ because this would violate CF. If $R_{i}\left(s^{*}\right) \neq \varnothing$ and $\pi_{i} \in R_{i}\left(s^{*}\right)$ then $u_{i \pi_{i}}\left(s_{\pi_{i}}^{*}\right) \geq u_{i j}\left(s_{j}^{*}\right)$ for all $j \in N \cup\{0\}$. Hence, $\left(\pi, s^{*}\right)$ is $\mathrm{CF}$, so $\pi$ is compatible with $s^{*}$.

However, not all assignments that are compatible with $s^{*}$ are desirable from an efficiency perspective, since for some of them it may well be the case that at least one worker can be made better off without hurting any other worker and without increasing the wages. This is illustrated in the following example.

Example 2. Suppose that $M=\{1,2,3,4\}$ and $N=\{1,2,3\}$ so one of the workers will be unemployed. Assume next that the government announces that the unemployment benefit $s_{0}$ equals 1 and that the minimum wages are given by $w=\left(w_{1}, w_{2}, w_{3}\right)=(2,2,2)$. Let the preferences of the workers be represented by quasi-linear utility functions of the type $u_{i j}\left(s_{j}\right)=v_{i j}+s_{j}$ for $i \in M$ and $j \in N$ and the values of jobs to the workers be given by the matrix:

$$
\left[\begin{array}{llll}
v_{10} & v_{11} & v_{12} & v_{13} \\
v_{20} & v_{21} & v_{22} & v_{23} \\
v_{30} & v_{31} & v_{32} & v_{33} \\
v_{40} & v_{41} & v_{42} & v_{43}
\end{array}\right]=\left[\begin{array}{llll}
5 & 3 & 3 & 2 \\
3 & 4 & 2 & 5 \\
3 & 1 & 1 & 5 \\
5 & 2 & 3 & 2
\end{array}\right]
$$

In this case, it can be shown that the CF and minimal compensation vector is given by $s^{*}=(3,3,2)$, see Example 4. Moreover, from the quasi-linear specification, the above matrix and $s^{*}$ it follows directly that $R_{1}\left(s^{*}\right)=\{1,2\}, R_{2}\left(s^{*}\right)=\{1,3\}, R_{3}\left(s^{*}\right)=\{3\}$ and $R_{4}\left(s^{*}\right)=\{2\}$. Hence, there are four assignments that are compatible with $s^{*}$ by Lemma 3 namely:

$$
\begin{aligned}
& \pi=\left(\pi_{1}, \pi_{2}, \pi_{3}, \pi_{4}\right)=(1,0,3,2), \\
& \dot{\pi}=\left(\dot{\pi}_{1}, \dot{\pi}_{2}, \dot{\pi}_{3}, \dot{\pi}_{4}\right)=(1,3,0,2), \\
& \bar{\pi}=\left(\bar{\pi}_{1}, \bar{\pi}_{2}, \bar{\pi}_{3}, \bar{\pi}_{4}\right)=(2,1,3,0), \\
& \tilde{\pi}=\left(\tilde{\pi}_{1}, \tilde{\pi}_{2}, \tilde{\pi}_{3}, \tilde{\pi}_{4}\right)=(0,1,3,2) .
\end{aligned}
$$


However, $\hat{R}_{1}\left(s^{*}\right)=\{0,1,2\}$ and $\hat{R}_{4}\left(s^{*}\right)=\{0,2\}$ because:

$$
\begin{aligned}
& u_{10}\left(s_{0}\right)=u_{11}\left(s_{1}^{*}\right)=u_{12}\left(s_{2}^{*}\right)=6, \\
& u_{40}\left(s_{0}\right)=u_{42}\left(s_{2}^{*}\right)=6 .
\end{aligned}
$$

Note also that $\hat{R}_{2}\left(s^{*}\right)=R_{2}\left(s^{*}\right)$ and $\hat{R}_{3}\left(s^{*}\right)=R_{3}\left(s^{*}\right)$. It is now clear that allocation $\left(\pi, s^{*}\right)$ is not desirable from an efficiency point of view because worker 2 is strictly better off at allocation $\left(\bar{\pi}, s^{*}\right)$ and the other three workers gets the same utility. Allocation $\left(\dot{\pi}, s^{*}\right)$ is also disqualified since worker 3 is strictly better of at allocation $\left(\tilde{\pi}, s^{*}\right)$ and the remaining three workers are indifferent. Hence, not all assignments that are compatible with $s^{*}$ are desirable.

Example 2 illustrates that unless some efficiency restriction is imposed on the allocation, non-desirable outcomes may be selected by the employer. However, additional restrictions cannot be imposed on the compensation vector for at least two reasons. First, if wages are allowed to exceed the CF and minimal wages, then the cost-minimizing employer will be strictly worse off because the cost of hiring workers will increase. This is certainly not desirable from the viewpoint of the employer. Second, even if wages are allowed to be higher than the $\mathrm{CF}$ and minimal wages $s^{*}$ then there always exists another CF compensation vector where the compensation for each employed worker is strictly higher by Lemma 1 Then because the wages are not bounded from above it is always possible to make the employed workers better off. Hence, by allowing the wages to deviate from the $\mathrm{CF}$ and minimal compensations the general idea behind the concept of $\mathrm{CF}$ is undermined (see Section 7 for a further discussion). For these reasons, additional restriction must be imposed on the assignment and not on the compensation vector. Given this observation the following definition of efficiency is very natural.

Definition 3. For a given profile $u \in \mathcal{U}$ and allocation $\left(\pi^{*}, s\right)$, the corresponding assignment $\pi^{*}$ is said to be object efficient if there is no other feasible assignment $\pi$ that is compatible with $s \in F(u)$ with the property that $u_{i \pi_{i}}\left(s_{\pi_{i}}\right) \geq u_{i \pi_{i}^{*}}\left(s_{\pi_{i}^{*}}\right)$ for each $i \in M$ with at least one strict inequality.

In Example 2 we have already seen that assignments $\pi$ and $\dot{\pi}$ are not object efficient, because they fail to satisfy the requirements in Definition 3 . We are now ready to define a CF and minimal allocation.

Definition 4. For a given profile $u \in \mathcal{U}$, allocation $\left(\pi^{*}, s^{*}\right)$ is said to be CF and minimal if (i) $s^{*} \in F(u)$ is minimal and (ii) $\pi^{*}$ is object efficient and compatible with $s^{*}$.

In Example 2] both $\left(\bar{\pi}, s^{*}\right)$ and $\left(\tilde{\pi}, s^{*}\right)$ are CF and minimal allocations. In general, we obtain the following existence result.

Theorem 2. For each profile $u \in \mathcal{U}$, there exists a $C F$ and minimal allocation $\left(\pi^{*}, s^{*}\right)$.

Proof. Because a CF and minimal compensation vector $s^{*}$ exists by Theorem 1 there exists a finite number of assignments that are compatible with $s^{*}$. Clearly, there is at least one assignment that is object efficient and compatible with $s^{*}$.

Remark 1. Efficiency in this paper is defined in terms of object efficiency. Of course, Pareto efficiency also requires that an upper bound on the use of resources for compensations are explicitly added to the model. However, in some cases there may be a conflict between constrained fairness and object efficiency (see Example 2), a conflict that does not prevail between fairness and object efficiency. It is well-known that a fair assignment of indivisible objects is always object efficient, see e.g. Svensson (1983). In our model with constrained fairness, the conflict can only emerge at utility profiles where there is an employed worker that is indifferent to being unemployed at the given compensations. Thus, for almost all profiles, object efficiency is implied also by constrained fairness. 
Remark 2. Our model reflects a situation where the government's concern for welfare can be expressed only by fixing the minimum wage rates and the unemployment benefit. If it is possible for the government to make interpersonal utility comparisons, other means may be available, e.g., force firms to set wages not only so that equilibrium on the market for employed workers prevails but also so that the wages maximize some kind of social welfare function. See Section 7 for a further discussion.

\section{Strategic Properties}

This section investigates strategic properties of an allocation mechanism that always selects CF and minimal allocations. Such an allocation mechanism will be called the CF and minimal allocation (CFMA) mechanism and denoted by $\varphi^{C F}$. We will investigate under what circumstances the CFMA mechanism can or cannot be manipulated by a worker or a group of workers. As established in Theorem 1 , the CF and minimal compensation vector is unique but this does not necessarily mean that there is a unique CF and minimal allocation (in Example 2 for instance both assignments $\bar{\pi}$ and $\tilde{\pi}$ are object efficient and compatible with the $\mathrm{CF}$ and minimal compensation vector). This also means that the $\mathrm{CF}$ and minimal allocation mechanism $\varphi^{C F}$ may be a correspondence. More explicitly, for all profiles $u \in \mathcal{U}$, the outcome of the CFMA mechanism $\varphi^{C F}(u)$ is a non-empty set such that:

$$
\varphi^{C F}(u) \subseteq\{(\pi, s) \in \mathcal{A} \mid \text { allocation }(\pi, s) \text { is } \mathrm{CF} \text { and minimal }\} .
$$

The correspondence $\varphi^{C F}$ defined in this way is an allocation mechanism whenever $\varphi^{C F}$ is essentially singlevalued (ESV), i.e., whenever each agent is indifferent among the various outcomes that the allocation mechanism may have. The following notion of manipulability will be employed.

Definition 5. A mechanism $\varphi$ is manipulable at a profile $u \in \mathcal{U}$ by a group $C \subseteq N$ if there is a profile $v \in \mathcal{U}$ and two allocations $(\pi, s) \in \varphi(u)$ and $(\sigma, t) \in \varphi\left(v_{C}, u_{-C}\right)$ such that $u_{i \sigma_{i}}\left(t_{\sigma_{i}}\right)>u_{i \pi_{i}}\left(s_{\pi_{i}}\right)$ for all $i \in C$. If it is not possible for any group $C \subseteq M$ to manipulate the mechanism at profile $u \in \mathcal{U}$ it is said to be non-manipulable at profile $u \in \mathcal{U}$.

Non-manipulability is a demanding requirement and, as a consequence, many economic problems cannot be expected to be solved by a non-manipulable allocation mechanism (at least not for all preference profiles). This is also the case for the CFMA mechanism as illustrated in the following example.

Example 3. Suppose that $M=\{1,2\}$ and $N=\{1\}$ so one of the workers will be unemployed. Assume next that the workers are endowed with quasi-linear utility functions of the type $u_{i j}\left(s_{j}\right)=v_{i j}+s_{j}$ for $i \in M$ and $j \in N$. Let also $v_{10}=2, v_{11}=1, v_{20}=3, v_{21}=0, s_{0}=0$ and $w_{1}=1$. In this case it is easy to see that the CF and minimal compensation for job 1 is $s_{1}^{*}=w_{1}=1$ and that $\left(\pi_{1}, \pi_{2}\right)=(1,0)$. However, worker 1 can manipulate the mechanism by reporting $\hat{v}_{10}=3$ instead of $v_{10}=2$, because in this case worker 1 is still assigned job 1 but the CF and minimal compensation is now $\hat{s}_{1}^{*}=2>1$. So worker 1 can actually gain by misreporting.

However, we will demonstrate that even though the CFMA mechanism can be manipulated (as demonstrated in Example 3), this is not always the case. In fact, the possibility for a specific worker or a specific group of workers to manipulate the CFMA mechanism depends heavily on (i) if they are assigned a job or not and (ii) the relation between the minimum wages and the unemployment benefit.

Our first result regarding non-manipulability (Theorem 3, below) demonstrates that if the outcome of the CFMA mechanism is fair for the true preferences then it is not possible for any group of unemployed workers to manipulate the outcome of the mechanism. To get some intuition of this result, note that fair allocations 
will only be selected by the CFMA mechanism when the unemployment benefit is high relative to the minimum wages, i.e., when all unemployed workers actually prefer being unemployed rather than having a job. Hence, when the unemployment benefit is "high", the unemployed workers will not try to change the outcome of the CFMA mechanism because if they do, they risk ending up with a job at a (weakly) lower wage. Then since they prefer being unemployed at the given wages they certainly prefer being unemployed when the wages (weakly) decreases. The reason that the wage may weakly decrease is that if an unemployed worker falsely signals that he is interested in a job, the worker signals to the employer that the job is attractive. Then because the employer selects minimal wages where fairness is respected among the employed workers the employer can (weakly) reduce the wage and at the same time guarantee that fairness is respected among employed workers. Consequently, it is impossible for any group of unemployed workers to manipulate the outcome of the CFMA mechanism in their advantage when the outcome is fair for the true preferences. Before stating this result formally, we provide a key lemma that will be important in some of the subsequent proofs.

Lemma 4. Let $(\pi, s) \in \varphi^{C F}(u)$ and consider the non-empty subset $C \subseteq M$. Suppose now that there is a profile $v \in \mathcal{U}$ such that $(\sigma, t) \in \varphi^{C F}\left(v_{C}, u_{-C}\right)$ and:

(i) $u_{i \pi_{i}}\left(s_{\pi_{i}}\right) \geq u_{i j}\left(s_{j}\right)$ for all $i \in C$ and all $j \in N$,

(ii) $u_{i \sigma_{i}}\left(t_{\sigma_{i}}\right)>u_{i \pi_{i}}\left(s_{\pi_{i}}\right)$ for all $i \in C$.

Let also $G=\left\{i \in M \mid t_{\sigma_{i}}<s_{\sigma_{i}}\right\}$. Then $C \cap G=\varnothing$, and $\pi_{i}=0$ if $i \in G$.

Proof. From assumptions (i) and (ii) it follows that $u_{i \sigma_{i}}\left(t_{\sigma_{i}}\right)>u_{i \pi_{i}}\left(s_{\pi_{i}}\right) \geq u_{i \sigma_{i}}\left(s_{\sigma_{i}}\right)$ for all $i \in C$. Consequently, $t_{\sigma_{i}}>s_{\sigma_{i}}$ for all $i \in C$ by monotonicity. Hence, $C \cap G=\varnothing$.

Assume now that there is a worker $i \in G$ with $\pi_{i} \neq 0$, and let $D=\left\{j \in N \mid t_{j}<s_{j}\right\}$. Note now that $0 \notin D$ since $t_{0}=s_{0}$, and that $D \neq \varnothing$ because $G \neq \varnothing$ by assumption. Moreover, for the worker $i \in G$ with $\pi_{i} \neq 0$ it is clear that:

$$
u_{i \pi_{i}}\left(s_{\pi_{i}}\right)>u_{i j}\left(s_{j}\right) \text { for all } j \in N \cup\{0\}-D .
$$

This follows from the following sequence of inequalities:

$$
\begin{aligned}
& u_{i \pi_{i}}\left(s_{\pi_{i}}\right) \geq u_{i \sigma_{i}}\left(s_{\sigma_{i}}\right) \text { by CF because } \pi_{i} \neq 0, \\
& u_{i \sigma_{i}}\left(s_{\sigma_{i}}\right)>u_{i \sigma_{i}}\left(t_{\sigma_{i}}\right) \text { by monotonicity since } \sigma_{i} \in D \text { and } i \notin C, \\
& u_{i \sigma_{i}}\left(t_{\sigma_{i}}\right) \geq u_{i j}\left(t_{j}\right) \text { for all } j \in N \text { by CF as } \sigma_{i} \neq 0 \text { when } \sigma_{i} \in D \text { and } i \notin C, \\
& u_{i j}\left(t_{j}\right) \geq u_{i j}\left(s_{j}\right) \text { by monotonicity when } j \in N \cup\{0\}-D .
\end{aligned}
$$

Let now $G^{\prime}=\left\{i \in G \mid \pi_{i} \neq 0\right\}$ and note that $G^{\prime} \neq \varnothing$ by assumption. Thus, if $i \in G^{\prime}$ then $\pi_{i} \in D$ by condition (2) and CF. Then, because of condition (2), we can apply Lemma1 to find a CF allocation $(\tau, r)$ such that $r \leq s$ and $r \neq s$. But this contradicts that $s$ is CF and minimal. Hence, $\pi_{i}=0$ if $i \in G$.

Theorem 3. Suppose that $(\pi, s) \in \varphi^{C F}(u)$ and that allocation $(\pi, s)$ is fair. Then it is not possible for any group of unemployed workers $C \subseteq M$ to manipulate $\varphi^{C F}$ at profile $u \in \mathcal{U}$.

Proof. Suppose that some group of unemployed workers $C \subseteq M$ can manipulate $\varphi^{C F}$ at the profile $u \in \mathcal{U}$. Then there is a profile $v \in \mathcal{U}$ and an allocation $(\sigma, t) \in \varphi^{C F}\left(v_{C}, u_{-C}\right)$ such that:

$$
u_{i \sigma_{i}}\left(t_{\sigma_{i}}\right)>u_{i \pi_{i}}\left(s_{\pi_{i}}\right) \text { for all } i \in C \text {. }
$$


Let also $D=\left\{j \in N \mid t_{j}<s_{j}\right\}$ and $G=\left\{i \in M \mid \sigma_{i} \in D\right\}$. Clearly, $C \cap G=\varnothing$ by Lemma4 Suppose now that $D \neq \varnothing$ and consider worker $i \in G$ where $\pi_{i}=0$ by Lemma44. But then $u_{i \pi_{i}}\left(t_{\pi_{i}}\right)=u_{i \pi_{i}}\left(s_{\pi_{i}}\right) \geq u_{i \sigma_{i}}\left(s_{\sigma_{i}}\right)>u_{i \sigma_{i}}\left(t_{\sigma_{i}}\right)$, by monotonicity, contradicting CF since $\sigma_{i}>0$. Hence, $D=\varnothing$. But if $D=\varnothing$, then $t \geq s$. However, $t$ cannot be CF and minimal for the profile $\left(v_{C}, u_{-C}\right) \in \mathcal{U}$ if $t \neq s$ because $s \in F\left(v_{C}, u_{-C}\right)$ since $u_{i \pi_{i}}\left(s_{\pi_{i}}\right) \geq u_{i j}\left(s_{j}\right)$ for all $i \in M-C$ and all $j \in N$, by fairness, and $\pi_{i}=0$ for all $i \in C$, by assumption. Thus, $t=s$. But then allocation $(\pi, s)$ cannot be fair according to condition (3) which contradicts the assumption in the theorem. Hence, it is not possible for any group of unemployed workers $C \subseteq M$ to manipulate $\varphi^{C F}$ at profile $u \in \mathcal{U}$.

Theorem 3 asserts that when the unemployment benefit is high relative to the minimum wages the unemployed workers cannot gain by acting strategically. However, when there is an converse relationship between the unemployment benefit and the minimum wages (i.e., when the minimum wages are high relative to the unemployment benefit) the CFMA mechanism cannot be manipulated by any group of employed workers. Intuitively the reason for this is that the only way for an employed worker to obtain a higher utility is to get a job at a higher wage (recall that each employed worker is always assigned his most preferred job so the wage must increase otherwise the utility cannot increase). In order to increase the wage for the job, the employed worker has to signal that he is not that interested in it, i.e., to try to make the job over-supplied. But if the employed worker is not interested in the job at the given wage then some unemployed worker may take the job immediately due to the "low" unemployment benefit. Hence, by signalling less interest in the job, the employed worker risks being unemployed and in this case the utility certainly cannot increase because each employed worker (at least weakly) prefers being employed at the given salary rather than being unemployed, by definition of CF.

The crucial factor for obtaining the non-manipulability result for a group of employed workers is that the minimum wages must be so high relative to the unemployment benefit that some unemployed worker always is willing to take a job at the given wages or, equivalently, if the employed worker $i$ (for some reason) drops out of the job-market then there is always some other worker $j$ that is willing to take his job at the given wage 6 In this case the allocation is said to be tight. Formally, tightness is defined as follows:

Definition 6. Let $M^{\prime} \subseteq M$ be a non-empty set of employed workers at the $C F$ allocation $(\pi, s)$. If there exists some other assignment $\hat{\pi}$ that is compatible with $s$ where $\hat{\pi}_{i}=0$ for all $i \in M^{\prime}$ then allocation $(\pi, s)$ is said to be tight for $M^{\prime}$.

A similar concept of tightness for Nash equilibrium is used in Dubey (1982) and Svensson (1991).

Theorem 4. Suppose that $(\pi, s) \in \varphi^{C F}(u)$ and that allocation $(\pi, s)$ is $C F$ and tight for some group $C \subseteq M$ of employed workers. Then it is not possible for the group of employed workers $C \subseteq M$ to manipulate $\varphi^{C F}$ at profile $u \in \mathcal{U}$.

Proof. Suppose that some group of employed workers $C \subseteq M$ can manipulate $\varphi^{C F}$ at the profile $u \in \mathcal{U}$. Then there is a profile $v \in \mathcal{U}$ and an allocation $(\sigma, t) \in \varphi^{C F}\left(v_{C}, u_{-C}\right)$ such that:

$$
u_{i \sigma_{i}}\left(t_{\sigma_{i}}\right)>u_{i \pi_{i}}\left(s_{\pi_{i}}\right) \text { for all } i \in C .
$$

Let also $D=\left\{j \in N \mid t_{j}<s_{j}\right\}$ and $G=\left\{i \in M \mid \sigma_{i} \in D\right\}$. We first demonstrate that $D=\varnothing$. For this purpose, suppose that $D \neq \varnothing$ and consider the compensation vector $r$ where $r_{j}=\min \left\{s_{j}, t_{j}\right\}$ for all $j \in N$. Then $r \leq s$ and $r \neq s$ since $D \neq \varnothing$. Let now $\tau$ be an assignment defined by $\tau_{i}=\sigma_{i}$ if $i \in G, \tau_{i}=0$ if $\pi_{i}=\sigma_{k}$ for some

\footnotetext{
${ }^{6}$ In Example 3 this is the case if $w_{1}=3$ because then worker 2 is indifferent between receiving job 1 and being unemployed.
} 
$k \in G$ and $\tau_{i}=\pi_{i}$ for the remaining workers. According to Lemma 4 $\pi_{i}=0$ for all $i \in G$. This means that all workers in $G$ are unemployed at $(\pi, s)$ but employed at assignment $\tau$. Now, since $C \cap G=\varnothing$, by Lemma4, it follows from $\mathrm{CF}$ and the construction of $r$ that the following holds for all $i \in G$ :

$$
u_{i \sigma_{i}}\left(t_{\sigma_{i}}\right) \geq u_{i j}\left(t_{j}\right) \text { for all } j \in N \text { and, hence, } u_{i \tau_{i}}\left(r_{\tau_{i}}\right) \geq u_{i j}\left(r_{j}\right) \text { for all } j \in N \text {. }
$$

The above condition shows that $r \in F(u)$, which contradicts that $s$ is CF and minimal. Hence, $D=\varnothing$.

Consider next the profile $\left(v_{C}, u_{-C}\right) \in \mathcal{U}$ and the compensation vector $t$. Since $D=\varnothing$, it is clear that $t \geq s$. Moreover, $(\sigma, t) \in \varphi^{C F}\left(v_{C}, u_{-C}\right)$, by assumption, and there is an assignment $\delta$ such that $s \in F(u)$ and $\delta_{i}=0$ if $i \in C$, by tightness. But then also $s \in F\left(v_{C}, u_{-C}\right)$. Consequently, $t=s$ because $t$ is $\mathrm{CF}$ and minimal at the profile $\left(v_{C}, u_{-C}\right) \in \mathcal{U}$. But $t=s$ is a contradiction to condition (4) and CF since $\pi_{i} \neq 0$ for all $i \in C$. Hence, it is not possible for the group of employed workers $C \subseteq M$ to manipulate $\varphi^{C F}$ at profile $u \in \mathcal{U}$.

We immediately obtain the following two corollaries to Theorem 4

Corollary 1. Suppose that $(\pi, s) \in \varphi^{C F}(u)$ and $u_{i \pi_{i}}\left(s_{\pi_{i}}\right) \leq u_{i j}\left(s_{j}\right)$ for some $i \in M$ with $\pi_{i}=0$ for each $j \in N$. Then the mechanism cannot be manipulated by any employed worker.

Corollary 2. Suppose that $(\pi, s) \in \varphi^{C F}(u),|M| \geq 2|N|$ and $u_{i \pi_{i}}\left(s_{\pi_{i}}\right) \leq u_{i j}\left(s_{j}\right)$ for all $j \in N$ and all $i \in M$ with $\pi_{i}=0$. Then the mechanism cannot be manipulated by any group of employed workers.

Corollary 1 states that if employed worker $j$ is weakly envied by unemployed worker $i$ then it is impossible for worker $j$ to gain by misrepresenting his preferences. The requirement in Corollary 2 is much stronger. It requires that there should be at least as many unemployed workers as jobs and in addition that each unemployed worker is willing to take any job at the given wages. If this is the case, then it is impossible for any group of employed workers to successfully manipulate the CFMA mechanism. This conclusion can also be reached for much weaker requirements as reported in Theorem 4, e.g., if each employed worker is weakly envied by some unemployed worker and if all unemployed workers weakly envy employed workers. Note also that by combining Theorems 3 and 4 it is in fact possible to find allocations and wage structures where it is impossible for any group of employed workers and any group of unemployed workers to manipulate the outcome of the CFMA mechanism (this is the case in the example in footnote 6). This is reported in the following corollary.

Corollary 3. Suppose that $(\pi, s) \in \varphi^{C F}(u),|M| \geq 2|N|$ and that allocation $(\pi, s)$ is fair and tight for each subset of employed workers. Then the mechanism cannot be manipulated by any group of employed workers or any group of unemployed workers.

In the above we demonstrated that if the unemployment benefit is high relative to the minimum wages the unemployed workers cannot gain by acting strategically, and similarly the employed workers cannot manipulate the CFMA mechanism when the relationship between minimum wages and the unemployment benefit is reversed. In some sense, this result might appear parallel to the classical result for the two-sided market models where it is possible to design a non-manipulable mechanism either for the buyers or for the sellers but not for both buyers and sellers simultaneously, see e.g., Demange and Gale (1985). In the current paper, we show that it is generally impossible to design a mechanism that can reconcile the incentives of all workers (both employed and unemployed). However, there is a marked difference between the classical result and ours in that for the classical models, both buyers and sellers are exogenously given, whereas in our model, both employed workers and unemployed are endogenously generated by the mechanism. This 
implies that it will be extremely hard for any worker to engage in manipulation, because he does not know whether he will be employed or not until the termination of the mechanism.

To sum up, the CFMA mechanism is not always manipulable even theoretically. There are also several additional reasons why it is hard to manipulate the mechanism in practice. First, because the number of workers and jobs usually is large in real life situations, it is difficult for any worker to collect the information of all other workers. Second, if the number of workers and jobs is large then it is also extremely difficult and complicated for a worker to figure out a winning strategy by manipulating the CFMA mechanism even if all other workers behave sincerely. Third, if the workers knew that a mechanism is manipulable then they would automatically protect their information very carefully.

\section{Identification of Constrainedly Fair and Minimal Allocations}

The existence of a CF and minimal allocation as established in Theorem 2 is a good starting point but a related important problem is of course how to identify such an allocation. This task is achieved next when we propose an algorithm for doing so. This algorithm consists of two parts: One that identifies the CF and minimal compensation vector and one that finds an assignment that is object efficient and compatible with it.

Theorem 2 is valid for a large class of preferences since it only requires that $u_{i j}\left(s_{j}\right)$ be continuous and strictly increasing in $s_{j}$. In the remaining part of the paper we restrict our attention to the smaller but important class of quasi-linear preferences 7 This means that each worker $i \in M$ places a monetary value $v_{i j}$ on each job $j \in N \cup\{0\}$ and that the preferences are represented by the utility function:

$$
u_{i j}\left(s_{j}\right)=v_{i j}+s_{j} \text { for all } i \in M \text { and all } j \in N \cup\{0\} .
$$

We also suppose that all $v_{i j}$ are integer-valued (since in reality no worker can specify a monetary value more closely than to the nearest penny or dollar). The set of profiles with quasi-linear utility functions having the above properties is denoted by $\mathcal{U}^{q}$. For simplicity, it is also assumed that the wages, the minimum wages and the unemployment benefit all are integer numbers. Given quasi-linearity we obtain the following corollary to Theorem 1 .

Corollary 4. For each profile $u \in \mathcal{U}^{q}$, the $C F$ and minimal compensation vector $s^{*}$ is integer-valued.

Proof. Let $N^{\prime}=\left\{j \in N \mid s_{j}^{*}\right.$ is not an integer $\}, M^{\prime}=\left\{i \in M \mid \pi_{i} \in N^{\prime}\right\}$ and suppose that $N^{\prime} \neq \varnothing$. Then:

$$
v_{i \pi_{i}}+s_{\pi_{i}}^{*}>v_{i j}+s_{j}^{*} \text { for all } i \in M^{\prime} \text { and all } j \in N \cup\{0\}-N^{\prime},
$$

since the reservation values $v_{i j}$ are integer-valued. Then $s^{\prime} \in F(u)$ if $s_{j}^{\prime}=s_{j}^{*}$ for all $j \in N-N^{\prime}$ and $s_{j}^{\prime}=s_{j}^{*}-\varepsilon$ for all $j \in N^{\prime}$ for a sufficiently small $\varepsilon>0$ because condition (5) still holds for such a small and equal decrease in the wage for each job in $N^{\prime}$. Note, finally, that $s_{j}^{\prime} \geq w_{j}$ for $j \in N^{\prime}$, since $w_{j}$ is an integer while $s_{j}^{*}$ is not. This shows that $s^{*}$ is not minimal, which is a contradiction. Hence, $N^{\prime}=\varnothing$.

The main idea for identifying the minimal compensation vector is to start with minimum compensations and then increase the compensations for the over-supplied sets of jobs (i.e., the sets of jobs where the number

\footnotetext{
${ }^{7}$ This class of preferences is often used in the mechanism design, see e.g., Crawford and Knoer (1981), Demange et al. (1986), Klijn (2000), Brams and Kilgour (2001), and Haake et al. (2002).
} 
of workers who demand a job in the set is less than the number of jobs in the set) until it is possible to identify a $\mathrm{CF}$ and minimal compensation vector. A crucial difference between this algorithm and the procedures in, e.g., Crawford and Knoer (1981), Demange et al. (1986) and Su (1999) is that we do not, and cannot, work with over-demanded jobs, because it may well be the case that a job is always over-demanded (e.g., job 1 in Example 1 when $s_{1}>2$, see footnote 4).

In order to describe the algorithm in detail, we first need to introduce some notation. Recall that for any subset of jobs $T \subseteq N$ and any given compensation vector $s$ the set of workers who demand a job in $T$ is denoted by $M(s, T)$. A set of jobs $T \subseteq N$ is said to be over-supplied at compensation vector $s$ if the number of workers who demand a job in $T$ is less than the number of jobs in $T$, i.e., if $|M(s, T)|<|T|$. By Lemma2 there can be no over-supplied set of jobs at a CF allocation. An over-supplied set $T$ of jobs in $N$ is said to be minimal if no subset of $T$ is over-supplied.

Algorithm 1. The compensation vector at Step $t$ is denoted by $s^{t}$ and the algorithm consists of the following steps:

Start. The jobs, the unemployment benefit and the minimum wages are made public. Then every worker $i \in M$ reports his integer value $v_{i j}$ over each job $j \in N \cup\{0\}$ to the employer. From now on workers are waiting for their assignments and compensations, but will not participate in the following process of wage adjustment.

Step 0. The employer starts with the minimum compensations for the jobs in $N$, i.e., $s_{j}^{0}=w_{j}$ for all $j \in N$.

Step t. At each Step $t \geq 1$, the employer computes the minimal over-supplied sets of jobs in $N$. If there is no minimal over-supplied set of jobs, then stop. Otherwise, choose a minimal over-supplied set $T$ of jobs and increase the compensation for each job in the set $T$ by one unit and keep the compensation for the other jobs unchanged and continue to Step $t+1$.

Note that it suffices to consider an increase by one unit in Step t of the algorithm because the CF and minimal compensation vector is integer-valued by Corollary 4 . However, the adjustment process could be speeded up by considering carefully selected increases of (say) dollars instead of increases by one dollar at a time (i.e., increases by one unit). To see this suppose that there is a unique minimal over-supplied set of jobs $\hat{T}$ at Step t of Algorithm 1 and note that this set will, by construction of the procedure, be minimal and over-supplied at least until some agent outside $M\left(s^{t}, \hat{T}\right)$ is indifferent to the jobs that he demands in $N-\hat{T}$ and some job in $\hat{T}$. Say that the wages for the jobs in $\hat{T}$ must be increased by 50 dollars before this occurs. Instead of increasing the wages in fifty separate steps (i.e., unit increases) the employer could simply calculate the minimal increase of the wages in $\hat{T}$ which makes some worker outside $M\left(s^{t}, \hat{T}\right)$ indifferent between his topitems in $N-\hat{T}$ and some job in $\hat{T}$ and then directly increase the wages for the objects in $\hat{T}$ with this amount (in this case 50 dollars).

We are now ready to present the following result which establishes the convergence of the algorithm.

Theorem 5. For each reported profile $u \in \mathcal{U}^{q}$, Algorithm 1 finds the unique $C F$ and minimal compensation vector in a finite number of steps.

Proof. Let $s$ and $s^{*}$ be the compensation vector generated by the algorithm and the CF and minimal compensation vector, respectively. Note that the existence of an integer valued vector $s^{*}$ is guaranteed by Theorem 1 and Corollary 4. Moreover, there is an assignment $\pi$ that is compatible with $s^{*}$ by definition of $\mathrm{CF}$. We need to demonstrate that $s^{t} \leq s^{*}$ at all possible steps $t$ because if this is the case then the algorithm 
converges. Moreover, if the algorithm stops at some Step $t$ and $s_{j}^{t}<s_{j}^{*}$ for some $j \in N$ then there are no over-supplied jobs. But this contradicts that $s^{*}$ is CF and minimal. Hence, if $s^{t} \leq s^{*}$ at all possible steps $t$ of the algorithm, then $s^{t}=s^{*}$ at the last step of the algorithm which proves the statement.

Suppose now that $s_{j}>s_{j}^{*}$ for some $j \in N$, and let $t$ be the last step of the algorithm where $s^{t} \leq s^{*}$. Such a step exists since $s^{0}=w \leq s^{*}$. This means that the compensation for each job in the minimal over-supplied set $T \subseteq N$ is increased by one unit, and that $|T|>\left|M\left(s^{t}, T\right)\right|$ because the jobs in $T$ are over-supplied. Let also:

$$
T^{\prime}=\left\{j \in T \mid s_{j}^{t}=s_{j}^{*}\right\}
$$

and note that $T^{\prime} \neq \varnothing$, by construction of the algorithm. We next demonstrate that (i) the $\left|T^{\prime}\right|>0$ number of jobs in $T^{\prime}$ must be assigned to $\left|T^{\prime}\right|$ number of workers in $M\left(s^{t}, T^{\prime}\right)$ at an assignment $\pi$ that is compatible to $s^{*}$ and that (ii) $T^{\prime} \neq T$. To see this, note first that all workers $i \in M-M\left(s^{t}, T^{\prime}\right)$ strictly prefers some other job $k \in N-T^{\prime}$ to any job $j \in T^{\prime}$, i.e.,

$$
v_{i k}+s_{k}^{t}>v_{i j}+s_{j}^{t}
$$

But $s_{j}^{t}=s_{j}^{*}$ for all $j \in T^{\prime}$ and $s_{j}^{t} \leq s_{j}^{*}$ for all $j \in N-T^{\prime}$. These observations in combination with the above condition give:

$$
v_{i k}+s_{k}^{*} \geq v_{i k}+s_{k}^{t}>v_{i j}+s_{j}^{t}=v_{i j}+s_{j}^{*}
$$

for some $k \in N-T^{\prime}$ and all $i \in M-M\left(s^{t}, T^{\prime}\right)$ and all $j \in T^{\prime}$. From condition (6) it is clear that if a job in $T^{\prime}$ is assigned to a worker in $M-M\left(s^{t}, T^{\prime}\right)$ then CF is violated and this cannot be the case because $\pi$ is compatible with the CF and minimal compensation vector $s^{*}$. Hence, the $\left|T^{\prime}\right|>0$ number of jobs in $T^{\prime}$ must be assigned to $\left|T^{\prime}\right|$ number of workers in $M\left(s^{t}, T^{\prime}\right)$ at an assignment $\pi$ that is compatible with $s^{*}$. This conclusion in combination with the facts that $s_{j}^{t}=s_{j}^{*}$ for all $j \in T^{\prime}$ and $s_{j}^{t} \leq s_{j}^{*}$ for all $j \in N-T^{\prime}$ yield:

$$
\left|M\left(s^{t}, T^{\prime}\right)\right| \geq\left|M\left(s^{*}, T^{\prime}\right)\right| .
$$

Suppose now that $T=T^{\prime}$. Since $|T|>\left|M\left(s^{t}, T\right)\right|$, by assumption, it follows from condition (7) that:

$$
|T|>\left|M\left(s^{t}, T\right)\right| \geq\left|M\left(s^{*}, T\right)\right| .
$$

But this means that the jobs in $T$ are over-supplied at $s^{*}$, and this cannot be the case. Hence, $T \neq T^{\prime}$.

Collect now all workers $i \in M\left(s^{t}, T^{\prime}\right)$ where:

$$
v_{i k}+s_{k}^{t}>v_{i j}+s_{j}^{t} \text { for some } k \in T^{\prime} \text { and all } j \in T-T^{\prime},
$$

in the set $M^{\prime} \subseteq M\left(s^{t}, T^{\prime}\right)$. Since $s_{j}^{t}<s_{j}^{*}$ for all $j \in T-T^{\prime}$, it is clear that each worker in $M\left(s^{t}, T^{\prime}\right)$ that is assigned a job in $T^{\prime}$ must belong to $M^{\prime}$. But since all jobs in $T^{\prime}$ must be assigned to some worker in $M\left(s^{t}, T^{\prime}\right)$, it follows that $\left|M^{\prime}\right| \geq\left|T^{\prime}\right|>0$. Hence, $M^{\prime} \neq \varnothing$. Note next that if $M^{\prime}=M\left(s^{t}, T\right)$, then all jobs in $T-T^{\prime} \neq \varnothing$ are over-supplied, which contradicts that $T$ is a minimal over-supplied set. Hence, $M^{\prime}$ is a non-empty and proper subset of $M\left(s^{t}, T\right)$. Note, finally, that $M\left(s^{t}, T\right)-M^{\prime}=M\left(s^{t}, T-T^{\prime}\right) \neq \varnothing$. The above facts, in combination with $|T|>\left|M\left(s^{t}, T\right)\right|>0$ and $0<\left|T^{\prime}\right| \leq\left|M^{\prime}\right|$, give:

$$
\left|T-T^{\prime}\right|>\left|M\left(s^{t}, T\right)-M^{\prime}\right|=\left|M\left(s^{t}, T-T^{\prime}\right)\right|>0,
$$

so $T-T^{\prime}$ is a non-empty and proper over-supplied subset of $T$, contradicting that $T$ is the minimal oversupplied set. Hence, $s^{t} \leq s^{*}$ at all steps $t$ of the algorithm.

It should be noted that Algorithm 1 works as long as $|N| \leq|M|$, i.e., its functionality is not dependent on the model assumption that $|N|<|M|$. This means that the algorithm can be adopted to identify the fair and 
minimal compensation vector when $|N|=|M|$ because in this bounding case a CF compensation vector is also a fair compensation vector (see footnote 5) 8 Consequently, if the government is the employer and the government finds it unacceptable that unemployed workers envy employed workers and in addition is willing to have flexible (and not fixed) unemployment benefits then the fair and minimal compensation vector can be identified even in the case when $|N|<|M|$ simply by adding $|M|-|N|>0$ dummy-objects to the set $N$ and then run Algorithm 1. We next illustrate the procedure with the aid of a simple numerical example.

Example 4. This example is based on the same premises as Example 2] i.e., $s_{0}=1, w=\left(w_{1}, w_{2}, w_{3}\right)=$ $(2,2,2)$ and the values of jobs to the workers are given by matrix (1). At Step 0, the compensations are given by $s^{0}=w=(2,2,2)$. Given the above information it is easy to calculate who demands a job in each of the six (non-empty) subsets of jobs in $N$. Consider, for example, the subset that consists only of job 1, i.e., $T=\{1\}$. It is clear that no worker demands this job at Step 0 because:

$$
\begin{aligned}
& v_{10}+s_{0}=6>5=v_{11}+s_{1}^{0}, \\
& v_{23}+s_{3}^{0}=7>6=v_{21}+s_{1}^{0}, \\
& v_{33}+s_{3}^{0}=7>3=v_{31}+s_{1}^{0}, \\
& v_{40}+s_{0}=6>4=v_{41}+s_{1}^{0} .
\end{aligned}
$$

Hence, $M\left(s^{0},\{1\}\right)=\varnothing$. In Table 1 , the set $M\left(s^{0}, T\right)$ is specified for each subset of jobs $T \subseteq N$ given compensation vector $s^{0}$ (see column 2). From the table, it is clear that there are two minimal over-supplied sets of jobs; $\{1\}$ and $\{2\}$. Hence, the compensation for job 1 or job 2 must be increased in Step 1 of the algorithm. Suppose that the compensation for job 1 is increased by one unit, so $s^{1}=(3,2,2)$. Now, the set $\{2\}$ is the minimal over-supplied set (see Table 1 , column 3$)$. This means that $s^{2}=(3,3,2)$. But then there are no over-supplied jobs (see Table 1, column 4). Hence, $s^{*}=s^{2}=(3,3,2)$.

Table 1: Illustration of the set $M\left(s^{t}, T\right)$ for each subset of jobs $T \subseteq N$ given compensation the vector $s^{t}$ from Step $t$ of Algorithm 1 (based on Example 4 .

\begin{tabular}{llll}
\hline$T$ & $M\left(s^{0}, T\right)$ & $M\left(s^{1}, T\right)$ & $M\left(s^{2}, T\right)$ \\
\hline$\{1\}$ & $\varnothing$ & $\{1,2\}$ & $\{1,2\}$ \\
$\{2\}$ & $\varnothing$ & $\varnothing$ & $\{1,4\}$ \\
$\{3\}$ & $\{2,3\}$ & $\{2,3\}$ & $\{2,3\}$ \\
$\{1,2\}$ & $\varnothing$ & $\{1,2\}$ & $\{1,2,4\}$ \\
$\{1,3\}$ & $\{2,3\}$ & $\{1,2,3\}$ & $\{1,2,3\}$ \\
$\{2,3\}$ & $\{2,3\}$ & $\{2,3\}$ & $M$ \\
$\{1,2,3\}$ & $\{2,3\}$ & $\{1,2,3\}$ & $M$ \\
\hline
\end{tabular}

Given that we now can identify the $\mathrm{CF}$ and minimal compensation vector $s^{*}$, it only remains to find a procedure to identify an object efficient assignment that is compatible with $s^{*}$ given quasi-linear preferences. The following lemma states a condition that can be used to identify an efficient allocation for a given CF and minimal compensation vector $s^{*}$. This condition is well-known and frequently adopted, see, e.g., Aragones (1995) or Klijn (2000).

\footnotetext{
${ }^{8}$ This key observation will also be useful in Section 7 (see in particular the proof of Theorem 6) when the social desirability of $\mathrm{CF}$ and minimal allocations is discussed.
} 
Lemma 5. If allocation $\left(\pi^{*}, s\right)$ is $C F$ and the following condition holds for all assignments $\pi$ that are compatible with $s$ :

$$
\sum_{i \in M} v_{i \pi_{i}^{*}} \geq \sum_{i \in M} v_{i \pi_{i}},
$$

then allocation $\left(\pi^{*}, s\right)$ is $C F$ and object efficient.

Proof. Suppose that allocation $\left(\pi^{*}, s\right)$ is CF and that condition $(8)$ holds but that allocation $\left(\pi^{*}, s\right)$ not is object efficient. Then there exists some other assignment $\pi$ that is compatible with $s$ with the property that $v_{i \pi_{i}^{*}}+s_{\pi_{i}^{*}} \leq v_{i \pi_{i}}+s_{\pi_{i}}$ for all $i \in M$ with at least one strict inequality by Definition 3 . By adding the $|M|$ conditions of this type the wages cancel out and we obtain:

$$
\sum_{i \in M} v_{i \pi_{i}^{*}}<\sum_{i \in M} v_{i \pi_{i}}
$$

which contradicts that condition (8) is satisfied. Hence, allocation $\left(\pi^{*}, s\right)$ is $\mathrm{CF}$ and object efficient.

Knowing that condition (8) is important for identifying object efficient assignments that are compatible with the $\mathrm{CF}$ and minimal compensation vector $s^{*}$ an important problem is how to identify assignments that satisfy condition (8). As it turns out, this problem reduces to a standard (linear) maximum weight matching problem of the type:

$$
\begin{array}{ll}
\max & \sum_{i \in N} \sum_{j \in M} \gamma_{i j} v_{i j} \\
\text { s.t. } & \sum_{j \in M} \gamma_{i j}=1 \text { for all } i \in N \\
& \sum_{i \in N} \gamma_{i j}=1 \text { for all } j \in M \\
& \gamma_{i j} \in\{0,1\} \text { if } j \in R_{i}\left(s^{*}\right) \text { and } \gamma_{i j}=0 \text { if } j \notin R_{i}\left(s^{*}\right)
\end{array}
$$

The solution to this problem identifies all assignments that are compatible with $s^{*}$ that satisfies condition (8). The interpretation of a solution vector $\gamma$ is that if $\gamma_{i j}=1$ then worker $i$ is assigned job $j$. Note also that because there is a finite number of assignments of jobs among workers, there will always exist at least one solution to the above problem but the solution vector $\gamma$ need not be unique (as illustrated in Example 2). Moreover, $R_{i}\left(s^{*}\right)$ is know from the last step of the $\mathrm{CF}$ and minimal compensation mechanism as demonstrated in Example 2. Hence, if $s^{*}$ is identified using Algorithm 1 it is not hard to find a compatible and object efficient assignment $\pi^{*}$. In fact, the following mechanism can be applied.

Algorithm 2. A CF and minimal allocation can be identified by adopting the followings three steps:

Start step. The employer identifies the CF and minimal compensation vector $s^{*}$ with the aid of Algorithm 1$]$

Intermediate Step. Given $s^{*}$ from the Start step the employer solves problem (9) in order to identify all assignments that are object efficient and compatible with $s^{*}$.

Final step. The employer selects one of the object efficient assignments from the Intermediate Step, say $\pi^{*}$, and assign job $\pi_{i}^{*}$ to worker $i \in M$ with salary $s_{\pi_{i}^{*}}^{*}$ 
By revisiting Examples 2 and 4, it is clear that two assignments pass the Intermediate Step, i.e., assignments $\bar{\pi}$ and $\tilde{\pi}$. Hence, the employer must apply some tie-breaking rule in the Final Step in order to determine the outcome. This tie-breaking rule can be based on a lottery, a priority list etc. Note however that in order for all strategic properties reported in Section 5 to hold this rule must be essentially single-valued. This will for example be the case when the employer ranks the workers in terms of labor market experience and selects the allocation from the Final Step which guarantees that the most experienced workers that demand a job (note that a worker demands a job at compensations $s^{*}$ when $R_{i}\left(s^{*}\right) \neq \varnothing$ ) also are assigned a job.

\section{Social Desirability}

The $\mathrm{CF}$ and minimal allocation mechanism is primarily intended for a cost-minimizing employer. This is also reflected in Definition 3 and Algorithm 2, where the CF and minimal allocation is found by first identifying the $\mathrm{CF}$ and minimal compensation vector $s^{*}$ and then by selecting an object efficient assignment that is compatible with $s^{*}$. However, from a social desirability perspective it is natural to consider allocations that maximize the sum of the values of the jobs to the workers. Note the key difference between the two: from the viewpoint of employers, the compensation vector is minimized first and then the job assignment is identified, but from the viewpoint of social desirability, the job assignment $\hat{\pi}$ is selected first and then, conditional on $\hat{\pi}$, the minimum salaries are identified. In this section, we describe how Algorithms 1 and 2 can be modified to achieve the social desirable outcome 9

As described above, the sum of the values of the jobs to the workers is maximized at the social desirable job allocation. This sum is maximized when problem (9) is solved but when the last constraint (i.e., $\gamma_{i j} \in$ $\{0,1\}$ if $j \in R_{i}\left(s^{*}\right)$ and $\gamma_{i j}=0$ if $\left.j \notin R_{i}\left(s^{*}\right)\right)$ is replaced by the constraint:

$$
\gamma_{i j} \in\{0,1\} \text { for all } i \in M \text { and all } j \in N
$$

This problem will be called the reduced problem (9), henceforth, and any assignment that solves the reduced problem (9) is called a welfare efficient assignment. Because the modified problem (9) is different from the maximization problem in the Intermediate Step of Algorithm 2 it is also clear that a CF and minimal job allocation need not be socially desirable. This is also demonstrated in the following example.

Example 5. Suppose that $M=\{1,2,3,4\}$ and $N=\{1,2,3\}$, so one of the workers will be unemployed. Assume also that $s_{0}=0$ and that $w=\left(w_{1}, w_{2}, w_{3}\right)=(1,0,7)$. Moreover, let the values of jobs to the workers be given by the matrix

$$
\left[\begin{array}{llll}
v_{10} & v_{11} & v_{12} & v_{13} \\
v_{20} & v_{21} & v_{22} & v_{23} \\
v_{30} & v_{31} & v_{32} & v_{33} \\
v_{40} & v_{41} & v_{42} & v_{43}
\end{array}\right]=\left[\begin{array}{llll}
6 & 8 & 12 & 7 \\
10 & 8 & 9 & 6 \\
8 & 7 & 7 & 2 \\
11 & 3 & 10 & 2
\end{array}\right]
$$

By running Algorithm 1, it is easy to confirm that the $\mathrm{CF}$ and minimal compensation vector is given by $s^{*}=(2,1,7)$ and that there is only one object efficient job assignment that is compatible with $s^{*}$, namely $\pi^{*}=\left(\pi_{1}^{*}, \pi_{2}^{*}, \pi_{3}^{*}, \pi_{4}^{*}\right)=(3,0,1,2)$. However, this assignment is not welfare efficient because $\sum_{i \in M} v_{\pi_{i}^{*}}=34$ and $\sum_{i \in M} v_{\hat{\pi}_{i}}=36$ for $\hat{\pi}=\left(\hat{\pi}_{1}, \hat{\pi}_{2}, \hat{\pi}_{3}, \hat{\pi}_{4}\right)=(2,3,1,0)$. Note also that, in this example, $\hat{\pi}$ is the unique welfare efficient assignment.

\footnotetext{
${ }^{9} \mathrm{We}$ wish to thank an anonymous referee for drawing our attention to the topics analyzed in this section.
} 
Example 5 5 reveals an important property of $\mathrm{CF}$ and minimal job allocations, namely, the cost of requiring salaries to be $\mathrm{CF}$ and minimal is that the resulting job assignment may not be welfare efficient. Suppose now that the employer identifies all job assignments that are object efficient and compatible with the $\mathrm{CF}$ and minimal compensation vector and realizes that none of them are welfare efficient. Assume further the employer believes that welfare efficiency is more important than cost minimization, for instance, if the employer is the government and therefore may have other objectives rather than cost minimization. Then, an alternative approach to find a job-allocation is to start by identifying a welfare efficient assignment and then to find a CF compensation vector that is compatible with this assignment and in addition minimizes the sum of the salaries. Such a job-allocation is called a CF and welfare efficient allocation and it is formally defined as follows.

Definition 7. For a given profile $u \in \mathcal{U}^{q}$, allocation $(\hat{\pi}, \hat{s})$ is said to be CF and welfare efficient if (i) $\hat{\pi}$ is welfare efficient and compatible with $\hat{s}$, (ii) $\hat{s} \in F(u)$ and (iii) $\sum_{i \in M} \hat{s}_{\hat{\pi}_{i}} \leq \sum_{i \in M} s_{\pi_{i}}$ for all $s \in F(u)$ that are compatible with some welfare efficient assignment $\pi$.

Before stating a concrete procedure for finding a $\mathrm{CF}$ and welfare efficient job assignment some notation must be introduced. For a given profile $u \in \mathcal{U}^{q}$, let all welfare efficient assignments be collected in the set $\mathcal{A}$, and for each $\pi \in \mathcal{A}$ let the employed workers be denoted by $E(\pi)$.

Algorithm 3. A CF and welfare efficient allocation can be identified by adopting the followings three steps:

Start step. The employer solves the reduced problem (9) to identify $\mathcal{A}$.

Intermediate step. For each $\pi \in \mathcal{A}$, identified in the Start step, the employer runs Algorithm $\square$ for the set of workers in $E(\pi)$.

Final step. Out of the $|\mathcal{A}|$ allocations identified in the Intermediate Step, the employer picks one that minimizes the sum of compensations, say $(\hat{\pi}, \hat{s})$, and assign job $\hat{\pi}_{i}$ to worker $i \in M$ with salary $\hat{s}_{\hat{\pi}_{i}}$.

Theorem 6. For each reported profile $u \in \mathcal{U}^{q}$, Algorithm 3 finds a $C F$ and welfare efficient allocation.

Proof. Note first that the welfare efficient assignments are identified in the Start step. In the Intermediate step, each $\pi \in \mathcal{A}$ is considered. More explicitly, for each $\pi \in \mathcal{A}$ the employer reduces the problem by not considering the workers in $M-E(\pi)$. In this reduced-form problem, there are exactly as many real jobs as there are workers, i.e. $n=|E(\pi)|$. The employer then applies Algorithm 1 to identify the CF and minimal salaries for the group of workers $E(\pi)$. Since there are exactly as many real jobs as there are workers in the reduced-form problem, two important conclusions can be drawn. First, Algorithm 1 converges in a finite number of steps by Theorem 5 (recall that the algorithm works as long as $n=|E(\pi)|$ ). Second, the $\mathrm{CF}$ and minimal compensation vector generated by Algorithm 1 for the reduced-form problem is compatible with $\pi$ because CF is equivalent to fairness when $n=|E(\pi)|$ (see footnote 5) and fairness implies welfare efficiency (see footnote 3). Hence, for each welfare efficient assignment $\pi \in \mathcal{A}$, the $\mathrm{CF}$ and minimal compensation vector can be identified for the reduced-form problem with $n=|E(\pi)|$ workers. Hence, if the employer, in the Final step, selects one of the $|\mathcal{A}|$ allocations identified in the Intermediate step that minimizes the sum of compensations, all requirements of Definition 7 are satisfied. Thus, the algorithm finds a CF and welfare efficient allocation.

Even though CF and welfare efficient allocations are desirable from a welfare perspective, they are not desirable from a cost-minimizing point of view. In fact, there is a potential trade-off between CF and minimal 
compensations and welfare efficiency. That is, if $s^{*}$ and $\hat{s}$ denote the CF and minimal compensation vector and a $\mathrm{CF}$ and welfare efficient compenstion vector, respectively, then, $\hat{s} \geq s^{*}$. This is an immediate consequence of Theorem 2] i.e., since $s^{*} \leq s$ for any $s \in F(u)$, it is clear that also $\hat{s} \geq s^{*}$. Consequently, if all CF and minimal job allocations not are welfare efficient, then the employer has to choose between " $\mathrm{CF}$ fair and minimal salaries" and "welfare efficiency". It is impossible to achieve both. This result is illustrated in the following example.

Example 6. This example is based on the same premisses as Example 5, i.e., $s_{0}=0, w=\left(w_{1}, w_{2}, w_{3}\right)=$ $(1,0,7)$ and the values of jobs to the workers be given by matrix (5). In Example 5] it was concluded that there is only one welfare efficient job assignment: $\hat{\pi}=\left(\hat{\pi}_{1}, \hat{\pi}_{2}, \hat{\pi}_{3}, \hat{\pi}_{4}\right)=(2,3,1,0)$. By letting $E(\hat{\pi})=\{1,2,3\}$ and by running Algorithm 1 for the reduced-form problem that only contains the workers in $E(\hat{\pi})$, the CF and welfare efficient compensation vector will be $\hat{s}=\left(\hat{s}_{1}, \hat{s}_{2}, \hat{s}_{3}\right)=(2,2,7)$. However, by running Algorithm 1 on the full problem with all $m$ workers, the resulting $\mathrm{CF}$ and minimal compensation vector is $s^{*}=\left(s_{1}^{*}, s_{2}^{*}, s_{3}^{*}\right)=(2,1,7)$. Hence, $\hat{s}_{j} \geq s_{j}^{*}$ for all $j \in N$ with strict inequality for $j=2$.

\section{References}

Alkan, A., Demange, G., Gale, D., 1991. Fair allocation of indivisible objects and criteria of justice. Econometrica 59, 1023-1039.

Andersson, T., Svensson, L-G., 2008. Weakly fair allocations and strategy-proofness. Rev. Econ. Design 11, 321-338.

Brams, S., Kilgour, M., 2001. Competitive fair division. J. Polit. Economy 109, 418-443.

Chaudhuri, 1986. Some implications of an intensity of measure. Soc. Choice Welfare 3, 255-270.

Crawford, V., Knoer, E., 1981. Job matching with heterogeneous firms and workers. Econometrica 49, 437-450.

Demange, G., Gale D., Sotomayor, M., 1986. Multi-item auctions. J. Polit. Economy 94, 863-872.

Diamantaras, D., Thomson, W., 1990. A refinement and extension of the no-envy concept. Econ. Letters 33, 217-222.

Dubey, P., 1982. Price-quantity strategic markets. Econometrica 50, 111-126.

Foley, D., 1967. Resource allocation and the public sector. Yale Econ. Essays 7, 43-98.

Haake, C-J., Raith, M., Su, F., 2003. Bidding for envy-freeness: a procedural approach to n-player fair division problems. Soc. Choice Welfare 19, 723-749.

Hall, P., 1935. On representatives of subsets. J. London Math. Society 10, 26-30.

Klijn, F., 2000. An algorithm for envy-free allocations in an economy with indivisible objects and money. Soc. Choice Welfare 17, 201-216.

Kuhn, H., 1955. The Hungarian method for the assignment problem. Naval Res. Logist. Quart. 2, 83-97.

Maskin, E., 1987. On the fair allocation of indivisible objects. In Feiwel, G. (Ed.), Arrow and the Foundations of the Theory of Economic Policy. New York University Press/New York, pp. 341-349.

Su, F., 1999. Rental harmony: Sperner's lemma in fair division. Amer. Math. Mon. 106, 930-942.

Svensson, L-G., 1983. Large indivisibilities: an analysis with respect to price equilibrium and fairness. Econometrica 51, 939-954.

Svensson, L-G., 1991. Nash implementation of competitive equilibria in a model with indivisible goods. Econometrica 59, 869-877.

Tadenuma, K., Thomson, W., 1991. No-envy and consistency in economies with indivisibilities. Econometrica $59,1755-1767$. 